\title{
Bifurcation of Parameter-Space and Chaos in Mira 2 Map
}

\section{Tao Jiang, Zhiyang Yang}

School of Information, Beijing Wuzi University, Beijing, China

Email: zyyang@amss.ac.cn, taojiang@amss.ac.cn

How to cite this paper: Jiang, T. and Yang, Z.Y. (2017) Bifurcation of Parameter-Space and Chaos in Mira 2 Map. Journal of Applied Mathematics and Physics, 5, 1899-1907. https://doi.org/10.4236/jamp.2017.59160

Received: August 4, 2017

Accepted: October 13, 2017

Published: October 16, 2017

\begin{abstract}
In this paper, we investigate Mira 2 map in parameter-space (A-B) and obtain some interesting dynamical behaviors. According to the parameter space of Mira 2 map, we take A and B as some groups of values and display complex

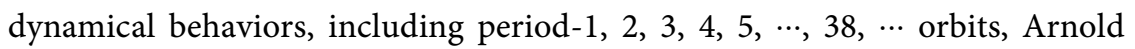
tongues observed in the circle map [7], crisis, some chaotic attractors, period-doubling bifurcation to chaos, quasi-period behaviors to chaos, chaos to quasi-period behaviors, bubble and onset of chaos.
\end{abstract}

\section{Keywords}

Mira 2 Map, Parameter-Space, Arnold Tongues

\section{Introduction}

Mira first introduced Mira 1 and 2 maps in [1], 1996. And in [2], Styness et al. attained a deeper understanding of the phenomenon-a transition from one chaos regime to another chaos regime via crisis-for $B$ falling in the interval $B_{c} \in[-2.0501226960083,-2.05012267960082]$ (where $B_{c}$ denotes the critical value of the parameter $B$ ) and other parameter $A=-1.5$.

Mira 2 map [1] has the functional form

$$
\left\{\begin{array}{l}
x_{n+1}=A x_{n}+y_{n} \\
y_{n+1} x_{n}^{2}+B
\end{array}\right.
$$

where $A$ and $B$ are real.

Though more dynamical behaviors of Mira 2 map (1) had gotten someone's less attention, we studied Mira 2 map and got many interesting dynamical behaviors, such as the conditions of the existence for fold bifurcation, flip bifurcation, Naimark-Sacker bifurcation and chaos in the sense of Marroto of this map in 
[3]. In this paper using numerical simulations [4], we obtained the distribution of dynamics in the parameter plane, the maximum Lyapunov exponent [5], fractal dimension [6] and more complex dynamical behaviors, including period-1, $2,3,4,5, \cdots, 38, \cdots$ orbits, Arnold tongues observed in the circle map [7], crisis, some chaotic attractors, period-doubling bifurcations to chaos, quasi-period behaviors to chaos, chaos to quasi-period behaviors, bubble, on set of chaos.

The paper is organized as follows. In Section 2, we give the parameter space of dynamical behaviors of Mira 2 map $(1)$ in $(A-B)$ plane. And in Section 3, the numerical simulations bifurcations in $(A-x)$ and $(B-x)$ planes for different values, the computation of maximum Lyapunov exponent corresponding to bifurcation diagram and the phase portraits at neighborhood of critical values are given.

\section{Bifurcation in the Parameter-Space}

In this section, we give the parameter space of dynamical behaviors of Mira 2 map (1) in $(A-B)$ plane.

In order to show more dynamics of Mira 2 map (1), we take $A$ and $B$ as the parameters and observe the motions of Mira 2 map (1) according to the initial condition $\left(x_{0}, y_{0}\right)=(0.001,0.05)$ of Mira 2 map (1). After computing some groups of the value scopes and the length of the grid of A and B, we find that there exist almost all dynamical motion of Mira 2 map (1) for the parameter interval $A \times B=[-2,2] \times[-4,0.5]$ and it takes relatively less time. The parameter-space of Mira 2 map (1) is shown in Figure 1. It is an isoperiodic diagram obtained by discretizing the parameter interval $A \times B=[-2,2] \times[-4,0.5]$ in a grid of $800 \times 900$ points equally spaced. This corresponds in Figure 1 to a same resolution in both $A$ and $B$ axes, that is $\Delta A=\Delta B=0.005$. For each point

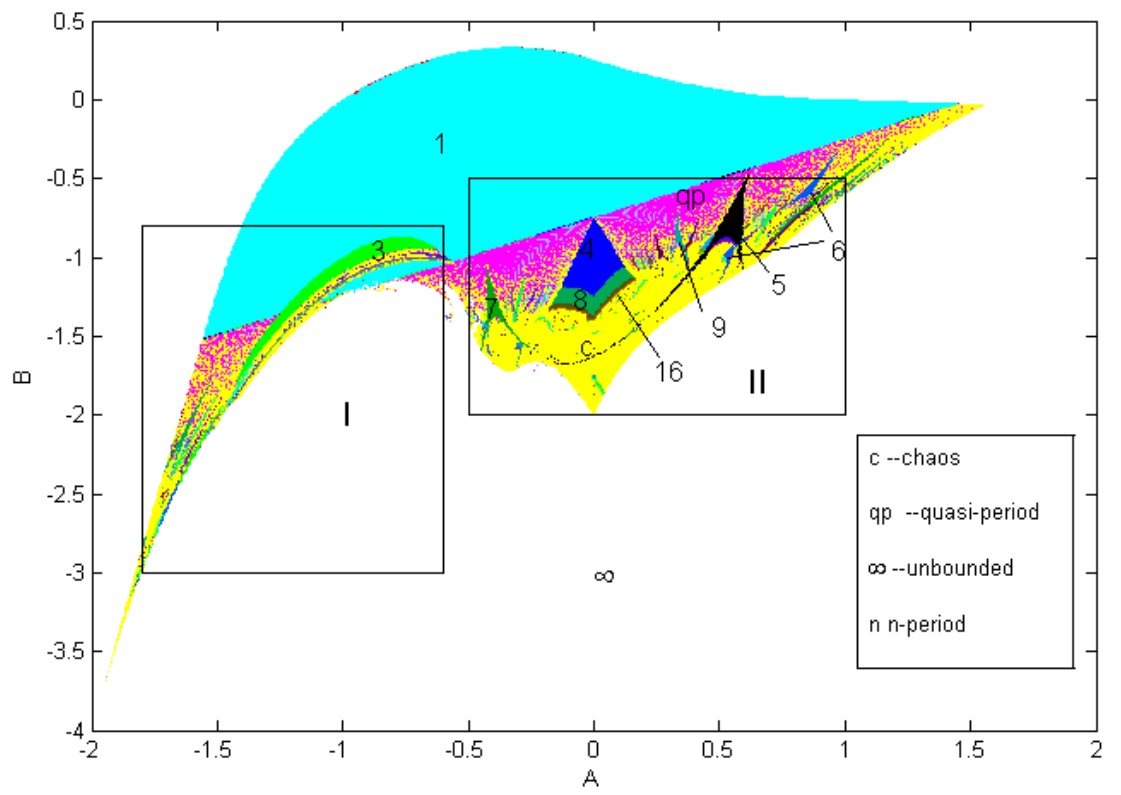

Figure 1. The parameter-space of Mira 2 map (1). 
$(A, B)$ in Figure 1, an orbit of initial condition $\left(x_{0}, y_{0}\right)=(0.001,0.05)$ converges to a chaotic attractor indicated by $c$, or to a quasi-periodic orbit indicated by $q p$, or to a n-period motion indicated by $n$, or to an attractor in infinity (unbounded attractor) indicated by $\infty$, after a transient of 5000 iterates.

In Figure 1, we can see quasiperiodic motion (purple region) is born exactly on the boundary-the line $B=\frac{A}{2}-\frac{3}{4}$-of period-1 (cyan) region, as a result of Naimark-Sacker bifurcations of period 1 (we give the condition of the existence of Naimark-Sacker bifurcation in [3]). There is a collection of periodic regions embedded in the quasiperiodic (purple) region not all of these observed clearly with the scale utilized in Figure 1. In two plots of Figure 2 one sees magnifications of the two regions inside of the boxes I and II of Figure 1, the first located in the range $-1.8 \leq a \leq-0.6,-3 \leq b \leq-0.8$, and the second in the range $-0.5 \leq a \leq 1,-2 \leq b \leq-0.5$. In Figure 2(a), period-1 (cyan) region and period-3 (green) region have well defined boundary. For parameter values taken along the boundary line, pitchfork bifurcation occurs, and parameter $b$ decreasing and passing through out period-3 (green) region Naimark-Sacker bifurcation occurs. In Figure 2(b), one sees periodic similar to the Arnold tongues observed immersed in purple region in the circle map [7].

\section{Bifurcation and Chaos in Numerical Simulations}

Now we present some numerical simulation results to show other interesting dynamical behaviors of Mira 2 map (1). According to the parameter space of Mira 2 map (1) in Figure 1, we take $A$ and $B$ as follows:

Case (1). Fixing $A=0$, and $-2 \leq B \leq-0.5$;

Case (2). Fixing $A=0.1$, and $-1.705 \leq B \leq 0.2$;

Case (3). Fixing $A=0.5$, and $-1.173 \leq B \leq-0.4$;

Case (4). Fixing $A=0.85$, and $-0.785 \leq B \leq-0.3$;

Case (5). Fixing $B=-2.2$, and $-1.682 \leq A \leq-1.57$;

For case (1) The bifurcation diagram of Mira 2 map (1) for $A=0$ in $(B, x)$ plane and the corresponding maximal Lyapunov exponents are given in Figure 3(a) and Figure 3(b), respectively. From Figure 3(a), we see period-doubling to

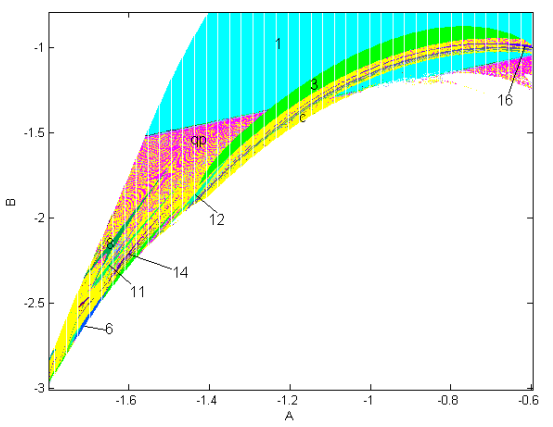

(a)

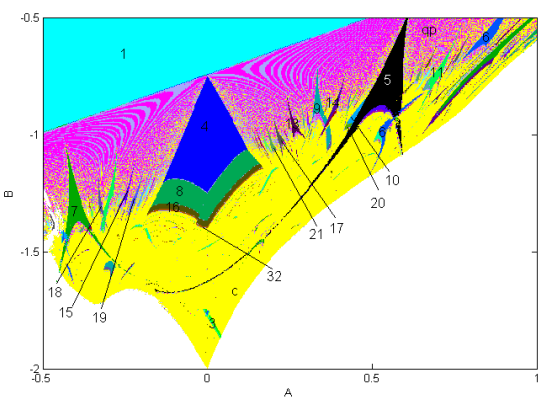

(b)

Figure 2. Magnification of the boxes (a) I, and (b) II in Figure 1. 


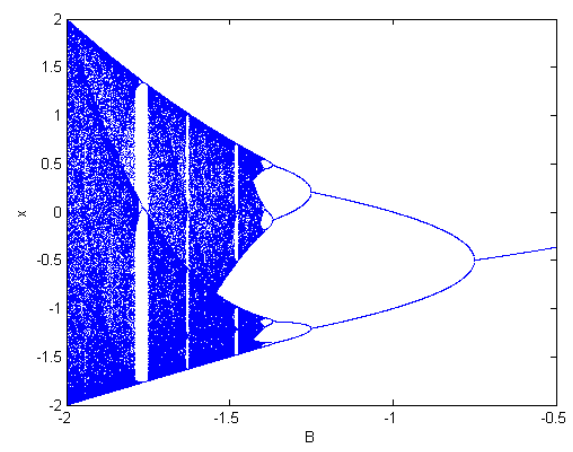

(a)

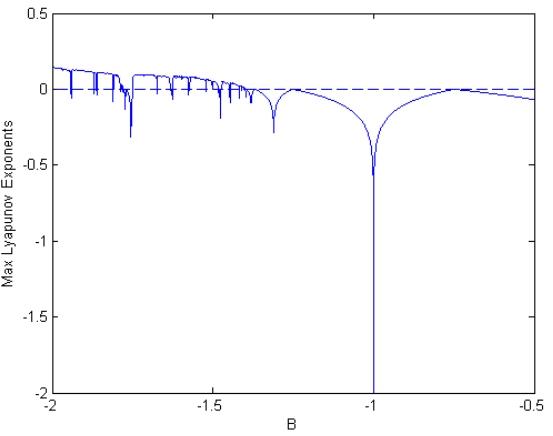

(b)

Figure 3. Bifurcation diagram and Lypunov exponents of Mira 2 map (1). Here $A=0$.

chaos occur with $B$ decreasing and chaos region abruptly disappears as $B=-1.4746,-1.6243,-1.749$, respectively. And when $B$ decrease to -2 , the chaos region turns to an attractor in infinity (unbounded attractor).

For case (2) The bifurcation diagram of Mira 2 map (1) for $A=0.1$ in $(B, x)$ plane and the corresponding maximal Lyapunov exponents are given in Figure 4(a) and Figure 4(b), respectively. In Figure 4(a), Mira 2 map (1) undergoes a Naimark-Sacker bifurcation from period-1 orbit at $B=-0.7$. At $B$ decreasing to $B=-1.0022$, quasi-period region suddenly disappears and six pieces of period-doubling to chaos occur. In the interval $B \in(-1.705,-1.22)$, period-doubling, Naimark-Sacker bifurcation and quasi-period behaviors are immersed in chaos region. The phase portraits of Mira 2 map (1) are shown in Figures 4(c)-(g), respectively. In Figures 4(c)-(e), the size of chaotic attractors at $B=-1.29$

(MaxLyapunovExponent $(M L E)=0.0394$, FractalDimension $(F D)=1.4692)$, $B=-1.34(M L E=0.0559, F D=2.2151), \quad$ and $\quad B=-1.6(M L E=0.0836)$, increases with $B$ decreasing. And the quasi-period orbits and its amplification are shown in Figure 4(f) and Figure 4(g), respectively.

For case (3) The bifurcation diagram of Mira 2 map (1) for $A=0.5$ in $(B, x)$ plane and the corresponding maximal Lyapunov exponents are given in Figure 5(a) and Figure 5(b), respectively. As $B$ decreasing, Mira 2 map (1) undergoes a Naimark-Sacker bifurcation from period-1 window at $B=\frac{A}{2}-\frac{3}{4}=-0.5$. At $B=-0.8025$, quasi-period region disappears to period-5 windows, and at $B=-0.8915$, period- 5 window becomes 15 period-doubling to chaos. Figures $\mathbf{5}(\mathrm{c})-(\mathrm{f})$ are shown chaotic attractors at

$B=-0.913,(M L E=0.0106, F D=1.0723)$, $B=-0.94,(M L E=0.0269, F D=1.2055), \quad B=-1,(M L E=0.045, F D=1.5670)$ and $B=-1.167(M L E=0.0845,1.5278)$, respectively.

For case (4) The bifurcation diagram of Mira 2 map (1) for $A=0.85$ in $(B, x)$ plane and the corresponding maximal Lyapunov exponents are given in Figure 6(a) and Figure 6(b), respectively. And the amplifications of (a) at $-0.665<B<-0.61$ and $-0.785<B<-0.68$ are shown in Figure 6(c) and 


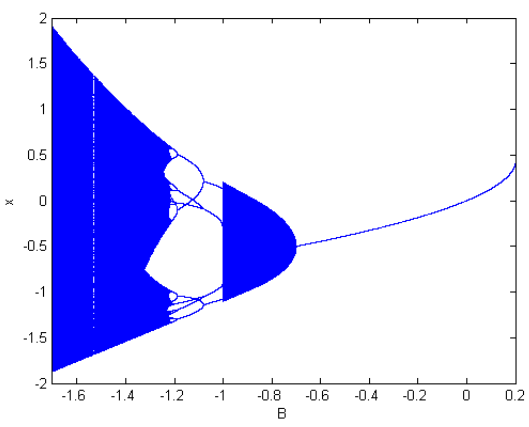

(a)

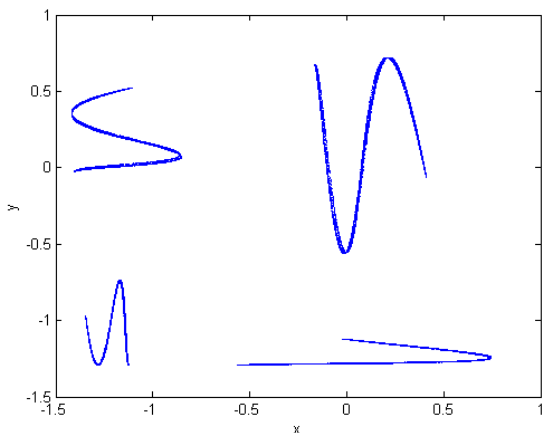

(c)

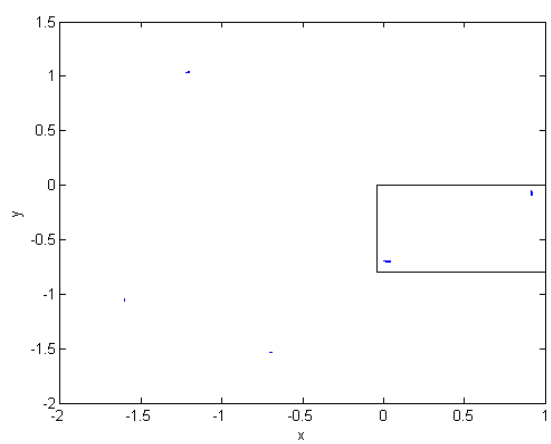

(e)

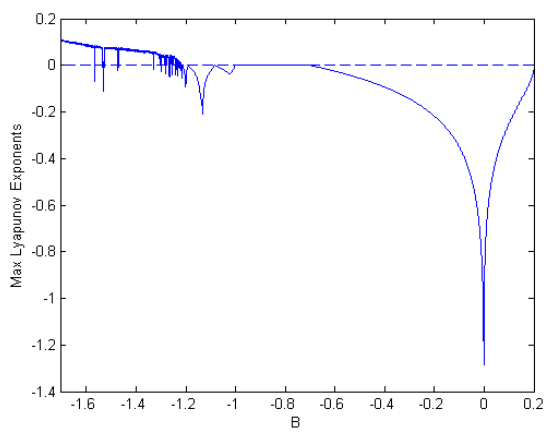

(b)

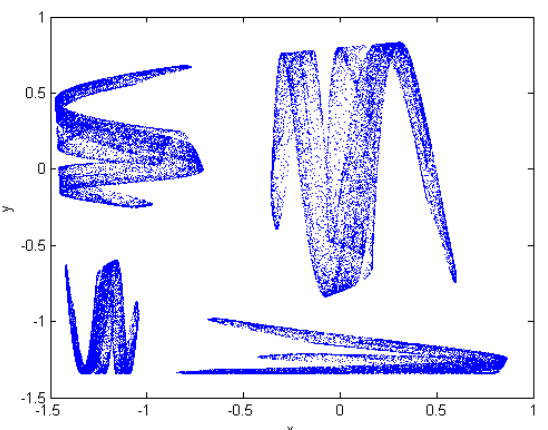

(d)

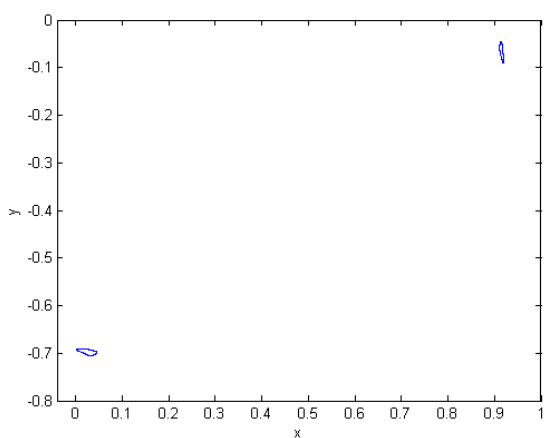

(f)

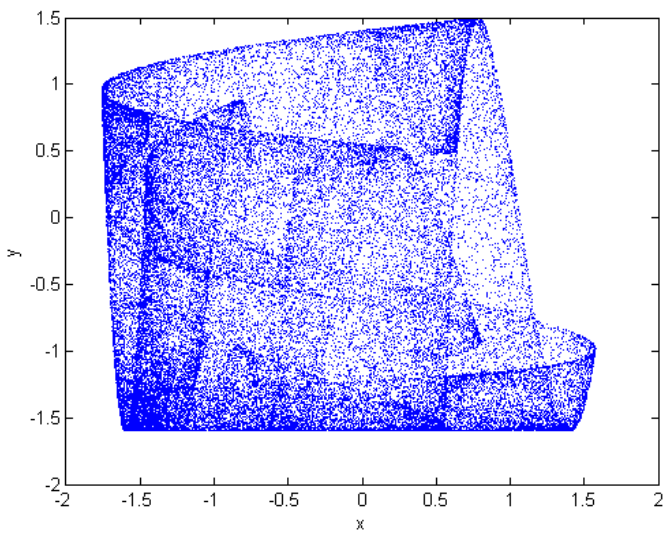

(g)

Figure 4. Bifurcation diagram and Lypunov exponents of Mira 2 map (1). Here $A=0.1$. (c)-(f) Phase portraits of Mira 2 map (1) at $B=-1.29, B=-1.34, B=-1.6$ and $B=-1.535$. (g) The amplification of ( $\mathrm{f}$ ). 


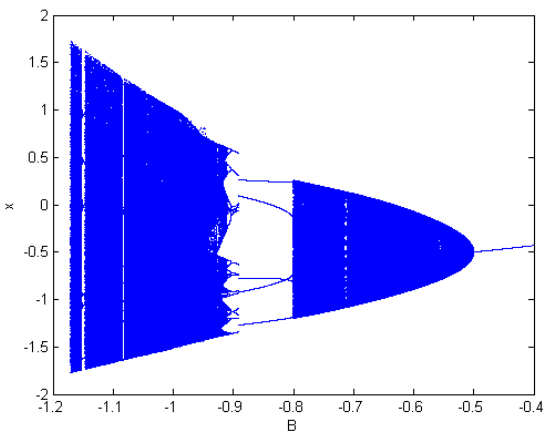

(a)

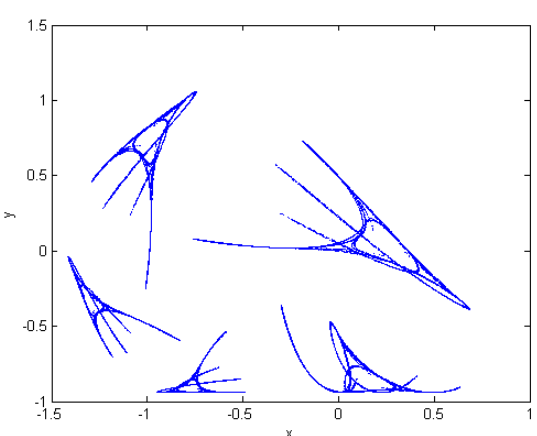

(c)

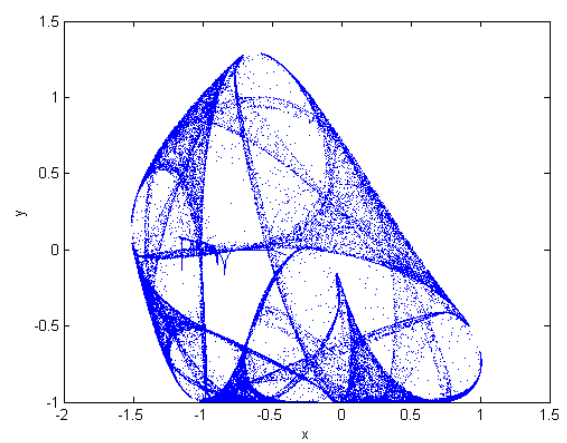

(e)

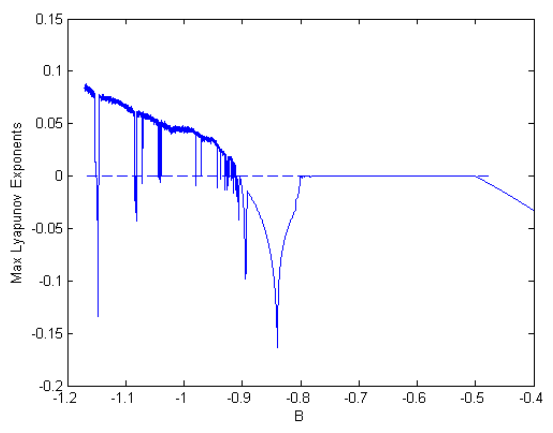

(b)

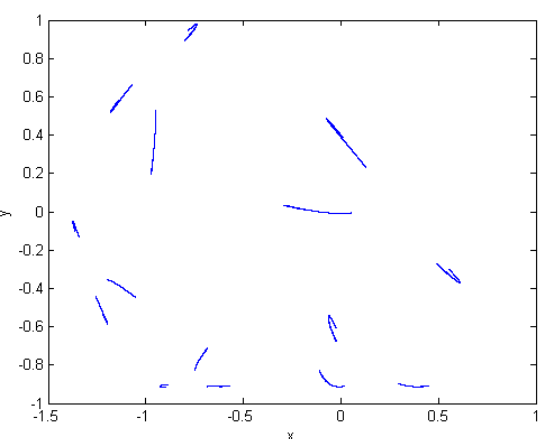

(d)

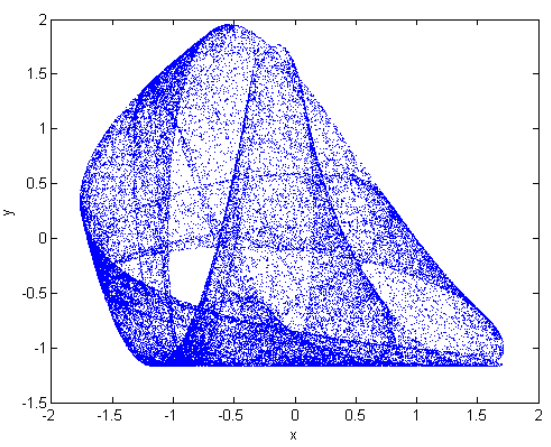

(f)

Figure 5. Bifurcation diagram and Lypunov exponents of Mira 2 map (1). Here $A=0.5$. (c)--(f) Phase portraits of Mira 2 map (1) at $B=-0.913, B=-0.94, B=-1$ and $B=-1.167$.

Figure 6(d), respectively. In Figure 6(a), Mira 2 map (1) undergoes a Naimark-Sacker bifurcation from period-1 window at $B=A / 2-3 / 4=-0.325$. As $B$ decreasing to $B=0.5535$, quasi-period behaviors suddenly disappear and period-6 window appears. In Figure 6(c), we observe that quasi-period behaviors and period windows alternatively appear, including period-18, 20, 21, 27, 28, 31, $33,43,53$, etc. And, as $B$ decreasing to $B=-0.6632,7$ pieces of inverse period-doubling to chaos appear, and in Figure 6(d), chaos region and period-doubling alternatively appear. The phase portraits of Mira 2 map (1) in Figures 6(e)-(i) are chaotic attractors at

$B=-0.6448(M L E=0.0047, F D=1.1148)$, 


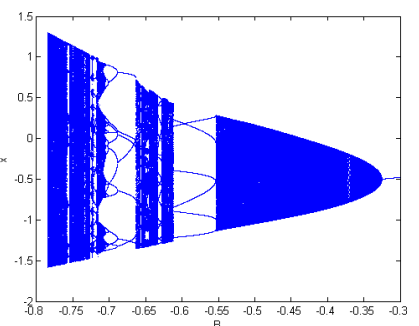

(a)

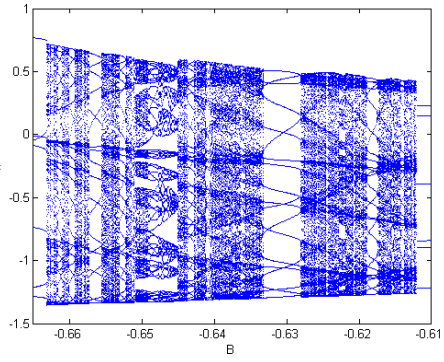

(c)

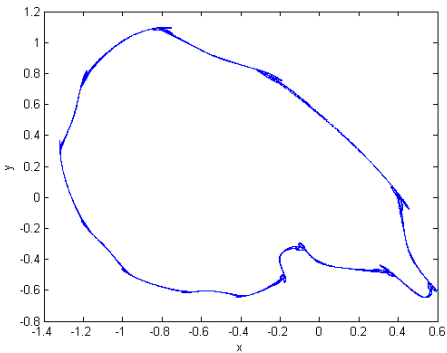

(e)

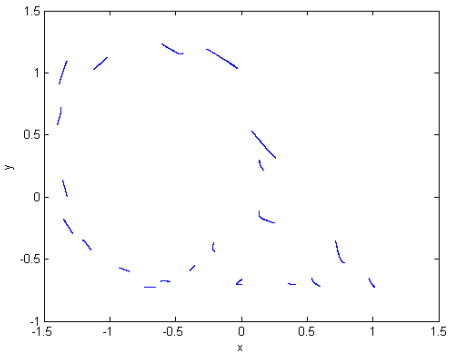

(g)

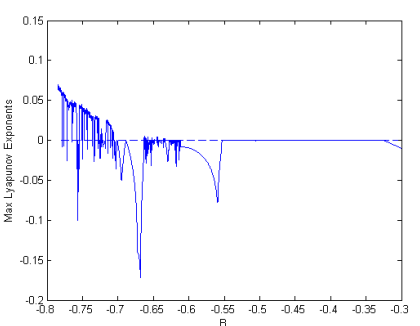

(b)

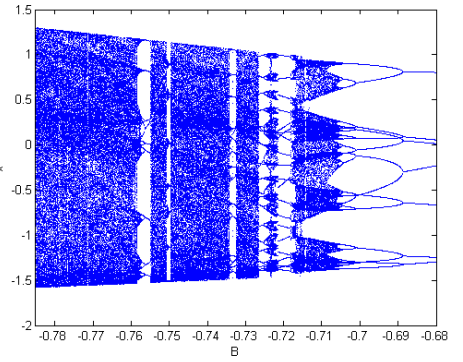

(d)

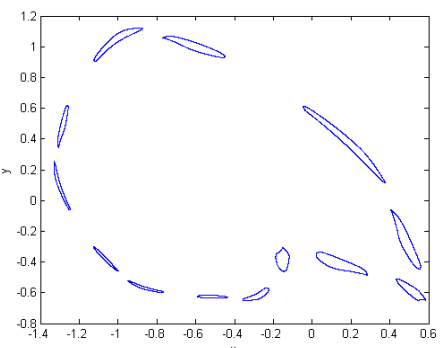

(f)

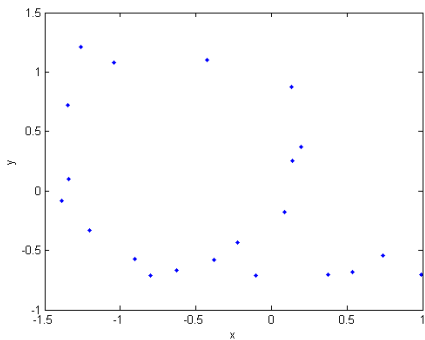

(h)

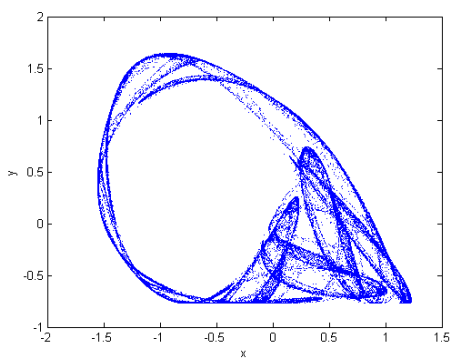

(i)

Figure 6. Bifurcation diagram and Lypunov exponents of Mira 2 map (1). Here $A=0.85$. (c) and (d) The amplification of (a). (e)-(i) Phase portraits of Mira 2 map (1) at $B=-0.6448, B=-0.649, B=-0.72, B=-0.724$ and $B=-0.77$. 
$B=-0.724(M L E=0.007, F D=1.0815)$ and

$B=-0.77(M L E=0.0423, F D=1.4178)$, quasi-period orbit at $B=-0.649$, and period- 21 orbit at $B=-0.72$, respectively.

For case (5) The bifurcation diagram of Mira 2 map (1) for $B=-2.2$ in $(A, x)$ plane and the corresponding maximal Lyapunov exponents are given in Figure 7(a) and Figure 7(c), respectively. As $A$ increasing to $A=-1.682$, the

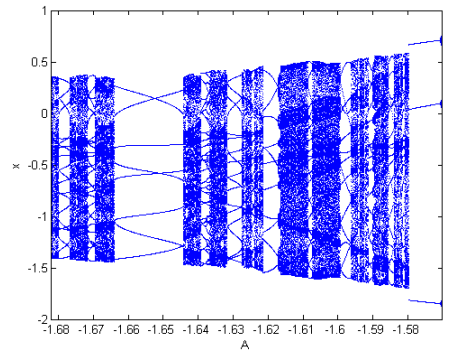

(a)

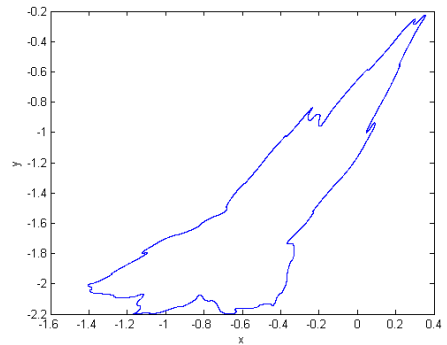

(c)

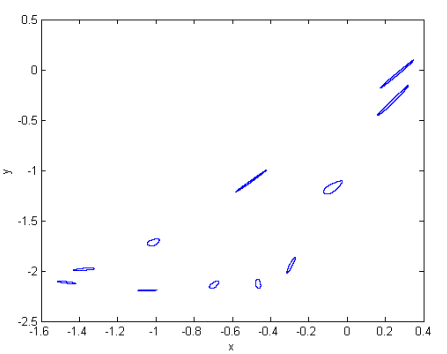

(e)

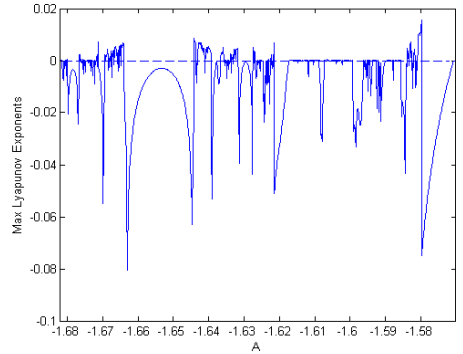

(b)

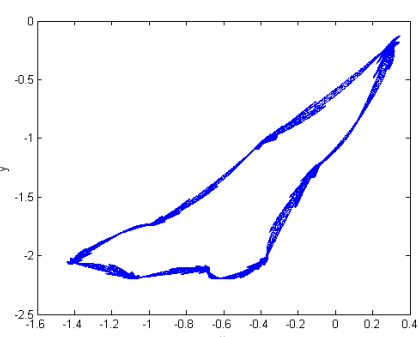

(d)

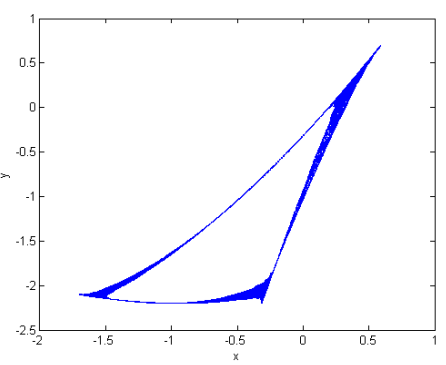

(f)

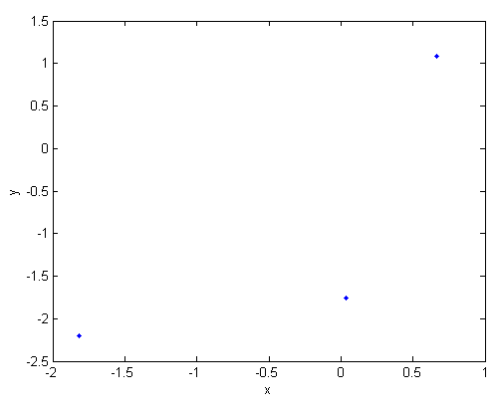

(g)

Figure 7. Bifurcation diagram and Lypunov exponents of Mira 2 map (1). Here $B=-2.2$. (c)-(g) Phase portraits of Mira 2 map (1) at $A=-1.6878, A=-1.665$, $A=-1.617, A=-1.5798$ and $A=-1.5797$. 
attractor in infinity suddenly converges to quasi-period orbit. And as $A$ increasing, quasi-period behaviors, period-orbits which include period-3, 8, 11, 17, 19, $20,21,25$, etc., and chaotic behaviors alternatively appear. When $A$ increase from $A=-1.5798$ to $A=-1.5797$, chaos disappears and period-3 orbit appear. We observe that 3 pieces of Naimark-Sacker bifurcation occur at $A=-1.5707$. As $A$ increasing to $A=-1.5707$, quasi-period behaviors suddenly disappear and the unbounded attractor appears. The phase portraits of quasi-period orbit, chaotic attractor, period-orbit of Mira 2 map (1) are shown in Figures 7(c)-(g) for $A=-1.6878, A=-1.665(M L E=0.0057, F D=1.1717)$, $A=-1.617, A=-1.5798(M L E=0.016, F D=1.1055)$ and $A=-1.5797$, respectively.

\section{Conclusion}

In this paper, we study Mira 2 map in parameter-space (A-B) and obtain some interesting dynamical behaviors. According to the parameter space of Mira 2 map, we take A and B as some groups of values and display complex dynamical behaviors.

\section{Acknowledgements}

This work was supported by the National Science Foundations of China (10671063 and 61571052).

\section{References}

[1] Mira, C., Barugola, A. and Gardini, L. (1996) Chaotic Dynamics in Two-Dimensional Nonvertible Map. World Scientific Publishing, Singapore. https://doi.org/10.1142/2252

[2] Styness, D., Hanan W.G., Pouryahya, S. and Herffernan, D.M. (2010) Scaling Relations and Critical Exponents for Two Dimensional Two Parameter Maps. The European Physical Journal B, 77, 469-478. https://doi.org/10.1140/epjb/e2010-00265-4

[3] Jiang, T. and Yang, Z.Y. (2017) Bifurcations and Chaos in Mira 2 Map. Acta Mathematicae Applicatae Sinica, English Series (Accepted).

[4] Nusse, H.E. and Yorke, J.A. (1997) Dynamics: Numerical Explorations. Springer, New York.

[5] Cartwright, J.H.E. (1999) Nonlinear Stiffness, Lyapunov Exponents, and Attractor Dimension. Physics Letters A, 26, 298-302. https://doi.org/10.1016/S0375-9601(99)00793-8

[6] Agiza, H.N., Elabbssy, E.M., El-Metwally, H. and Elsadany, A.A. (2009) Chaotic Dynamics of a Discrete Prey-Predator Model with Holling Type II. Nonlinear Analyse: Real World Application, 10, 116-129. https://doi.org/10.1016/j.nonrwa.2007.08.029

[7] Schuster, H.G. and Just, W. (2005) Deterministic Chaos: An Introduction. 4th Edition, Wiley-VCH Verlag GmbH \& Co., Weinheim.

https://doi.org/10.1002/3527604804 